\title{
든 REPTS
}

ISSN 1600-5368

\section{Crystal structure of $N^{\prime}$-hydroxy- pyrimidine-2-carboximidamide}

\section{Nithianantham Jeeva Jasmine, Packianathan Thomas Muthiah* and Nithianantham Stanley}

School of Chemistry, Bharathidasan University, Tiruchirappalli 620 024, Tamil Nadu, India. *Correspondence e-mail: tommtrichy@yahoo.co.in

Received 7 September 2014; accepted 9 September 2014

Edited by J. Simpson, University of Otago, New Zealand

The title compound, $\mathrm{C}_{5} \mathrm{H}_{6} \mathrm{~N}_{4} \mathrm{O}$, is approximately planar, with an angle of $11.04(15)^{\circ}$ between the planes of the pyrimidine ring and the non- $\mathrm{H}$ atoms of the carboximidamide unit. The molecule adopts an $E$ configuration about the $\mathrm{C}=\mathrm{N}$ double bond. In the crystal, adjacent molecules are linked by pairs of $\mathrm{N}-\mathrm{H} \cdots \mathrm{O}$ hydrogen bonds, forming inversion dimers with an $R_{2}^{2}(10)$ ring motif. The dimers are further linked via $\mathrm{N}-\mathrm{H} \cdots \mathrm{N}$ and $\mathrm{O}-\mathrm{H} \cdots \mathrm{N}$ hydrogen bonds into a sheet structure parallel to the $a c$ plane. The crystal structure also features $\mathrm{N}-\mathrm{H} \cdots \mathrm{O}$ and weak $\mathrm{C}-\mathrm{H}$...O hydrogen bonds and offset $\pi-\pi$ stacking interactions between adjacent pyrimidine rings [centroidcentroid distance $=3.622(1) \AA]$.

Keywords: crystal structure; pyrimidine-2-carboximidamide; non-covalent interactions; hydrogen bonding; $\pi-\pi$ stacking interactions; biological activity.

CCDC reference: 1018015

\section{Related literature}

For details of non-covalent interactions, see: Desiraju (2007). For the role of intermolecular hydrogen bonds in the design of organic crystals, see: Aakeroy \& Seddon (1993). For background to substituted $N^{\prime}$-hydroxybenzamidines as intermediates in the synthesis of 1,2,4-oxadiazole derivatives, see: Kundu et al. (2012). For the biological activity of substituted $N^{\prime}$-hydroxybenzamidines and 1,2,4-oxadiazole derivatives, see: Sakamoto et al. (2007); Tyrkov \& Sukhenko (2004).

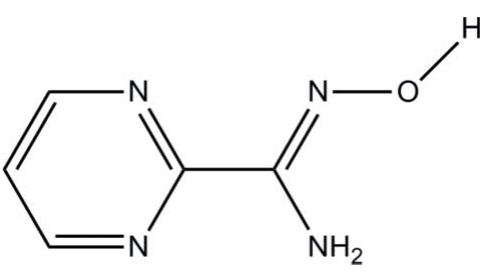

\section{Experimental}

\subsection{Crystal data}

$\mathrm{C}_{5} \mathrm{H}_{6} \mathrm{~N}_{4} \mathrm{O}$

$M_{r}=138.14$

Monoclinic, $P 2_{1} / c$

$a=7.4066$ (7) А

$b=8.0165(8) \AA$

$c=10.2200(9) \AA$

$\beta=101.888(6)^{\circ}$

$$
\begin{aligned}
& V=593.8(1) \AA^{3} \\
& Z=4 \\
& \text { Mo } K \alpha \text { radiation } \\
& \mu=0.12 \mathrm{~mm}^{-1} \\
& T=100 \mathrm{~K} \\
& 0.62 \times 0.17 \times 0.08 \mathrm{~mm}
\end{aligned}
$$

\subsection{Data collection}

Bruker SMART APEXII CCD area-detector diffractometer Absorption correction: multi-scan (SADABS; Bruker, 2009)

$T_{\min }=0.931, T_{\max }=0.990$

4073 measured reflections 1030 independent reflections 831 reflections with $I>2 \sigma(I)$ $R_{\text {int }}=0.047$

\subsection{Refinement}

$R\left[F^{2}>2 \sigma\left(F^{2}\right)\right]=0.054$

$w R\left(F^{2}\right)=0.181$

$S=1.13$

1030 reflections

103 parameters

$\mathrm{H}$ atoms treated by a mixture of independent and constrained refinement $\Delta \rho_{\max }=0.29{\mathrm{e} \AA^{-3}}^{-3}$ $\Delta \rho_{\min }=-0.31 \mathrm{e}^{-3}$

Table 1

Hydrogen-bond geometry $\left(\AA{ }^{\circ}\right)$.

\begin{tabular}{lllll}
\hline$D-\mathrm{H} \cdots A$ & $D-\mathrm{H}$ & $\mathrm{H} \cdots A$ & $D \cdots A$ & $D-\mathrm{H} \cdots A$ \\
\hline $\mathrm{N} 4-\mathrm{H} 2 N 4 \cdots \mathrm{O} 1^{\mathrm{i}}$ & $0.89(3)$ & $2.27(3)$ & $2.996(3)$ & $139(3)$ \\
$\mathrm{N} 4-\mathrm{H} 1 N 4 \cdots \mathrm{N} 3^{\mathrm{ii}}$ & $0.92(3)$ & $2.30(3)$ & $3.106(3)$ & $146(3)$ \\
$\mathrm{O} 1-\mathrm{H} 1 O 1 \cdots \mathrm{N} 2^{\mathrm{iii}}$ & $0.95(4)$ & $1.85(4)$ & $2.783(3)$ & $167(3)$ \\
$\mathrm{C} 3-\mathrm{H} 3 A \cdots \mathrm{O} 1^{\mathrm{iv}}$ & 0.95 & 2.51 & $3.305(4)$ & 141 \\
\hline
\end{tabular}

Symmetry codes: (i) $-x+2,-y+1,-z$; (ii) $x,-y+\frac{1}{2}, z-\frac{1}{2}$; (iii) $-x+2, y+\frac{1}{2},-z+\frac{1}{2}$; (iv) $x, y-1, z$

Data collection: APEX2 (Bruker, 2009); cell refinement: SAINT (Bruker, 2009); data reduction: SAINT; program(s) used to solve structure: SHELXTL (Sheldrick, 2008); program(s) used to refine structure: SHELXTL; molecular graphics: SHELXTL; software used to prepare material for publication: SHELXTL and PLATON (Spek, 2009).

\section{Acknowledgements}

NJJ thanks the UGC-SAP for the award of an RFSMS. PTM thanks the UGC for a one-time BSR grant.

Supporting information for this paper is available from the IUCr electronic archives (Reference: SJ5423). 


\section{data reports}

\section{References}

Aakeroy, C. B. \& Seddon, K. R. (1993). Chem. Soc. Rev. 22, 397-407. Bruker (2009). SADABS, APEX2 and SAINT. Bruker AXS Inc., Madison, Wisconsin, USA.

Desiraju, G. R. (2007). Angew. Chem. Int. Ed. 46, 8342-8356.

Kundu, M., Singh, B., Ghosh, T., Maiti, B. C. \& Maity, T. K. (2012). Indian J. Chem. Sect. B, 51, 493-497.
Sakamoto, T., Cullen, M. D., Hartman, T. L., Watson, K. M., Buckheit, R. W., Pannecouque, C., DeClercq, E. \& Cushman, M. (2007). J. Med. Chem. 50, 3314-3319.

Sheldrick, G. M. (2008). Acta Cryst. A64, 112-122.

Spek, A. L. (2009). Acta Cryst. D65, 148-155.

Tyrkov, A. G. \& Sukhenko, L. T. (2004). Pharm. Chem. J. 38, 30-38. 


\section{supporting information}

Acta Cryst. (2014). E70, o1107-o1108 [doi:10.1107/S1600536814020285]

\section{Crystal structure of $N^{\prime}$-hydroxypyrimidine-2-carboximidamide}

\section{Nithianantham Jeeva Jasmine, Packianathan Thomas Muthiah and Nithianantham Stanley}

\section{S1. Comment}

Supramolecular architectures assembled via various delicate noncovalent interactions such as hydrogen bonds, $\pi-\pi$ stacking and electrostatic interactions, have attracted intense interest in recent years because of their fascinating structural diversity and potential applications for functional materials (Desiraju, 2007). In particular, the application of intermolecular hydrogen bonding is a well known and efficient tool in the field of organic crystal design owing to their strength and directional properties (Aakeroy \& Seddon, 1993). Substituted $N^{\prime}$-hydroxybenzamidines are important intermediates obtained during the synthesis of pharmaceuticaly important 1,2,4-oxadiazole derivatives (Kundu et al., 2012). 1,2,4-oxadiazole derivatives are well known for their biological activities such as for anti-HIV (Sakamoto et al., 2007) and anti-microbial applications (Tyrkov \& Sukhenko, 2004). Herein, we report the crystal structure determination of the title compound, (I).

The asymmetric unit of the title compound is shown in Fig. 1. The essentially planar pyrimidine ring [N1/N2/C1-C4, maximum deviation of 0.009 (2) $\AA$ at atom C4] forms a dihedral angle of $11.04(15)^{\circ}$ with the hydroxyacetimidamide (N4/C5/N3/O1). The compound adopts an $E$ configuration across the $\mathrm{C} 5=\mathrm{N} 3$ double bond, as the $\mathrm{OH}$ group and benzene ring are on opposite sides of the double bond while the hydrogen atom of the hydroxy group is directed away from the $\mathrm{NH}_{2}$ group. The bond lengths and angles are within normal ranges.

In the crystal packing, molecules are linked by a pair of $\mathrm{N} 4-\mathrm{H} 2 \mathrm{~N} 4 \cdots \mathrm{O} 1^{i}$ hydrogen bonds (symmetry code in Table 1) into an inversion dimer, forming an $\mathrm{R}_{2}^{2}(10)$ ring motif. These molecules are self-assembled via $\mathrm{N} 4-\mathrm{H} 1 \mathrm{~N} 4 \cdots \mathrm{N} 3^{3 i}$ hydrogen bonds (graph-set notation C(4); symmetry code as in Table 1), which interconnect the dimers resulting in a sheet parallel to the $a c$ plane as shown in Fig 2. Furthermore, the crystal structure is stabilized by $\mathrm{O} 1-\mathrm{H} 1 \mathrm{O} 1 \cdots \mathrm{N} 2^{\mathrm{iii}}$ and weak $\mathrm{C} 3-\mathrm{H} 3 \mathrm{~A} \cdots \mathrm{O}^{\text {iv }}$ hydrogen bonds (symmetry code as in Table 1 ) and $\pi-\pi$ stacking interactions between the pyrimidine (N1/N2/C1-C4) rings [centroid-centroid distance = 3.622 (1) $\AA$; (symmetry code: $1-x,-y,-z)$ ].

\section{S2. Experimental}

A hot methanol solution ( $20 \mathrm{ml}$ ) of $N^{\prime}$-hydroxypyrimidine- 2-carboximidamide (69 mg, Aldrich) was warmed over a magnetic stirrer hotplate for a few minutes. The resulting solution was allowed to cool slowly at room temperature. Single crystals of the title compound (I) appeared from the mother liquor after a few days.

\section{S3. Refinement}

$\mathrm{O}$ - and $\mathrm{N}$-bound $\mathrm{H}$ atoms were located in a difference Fourier map and refined freely $[\mathrm{O}-\mathrm{H}=0.94$ (4) $\AA$ and $\mathrm{N}-\mathrm{H}=0.89$

(3) $\AA$ and 0.92 (3) $\AA]$. The remaining hydrogen atoms were positioned geometrically $[\mathrm{C}-\mathrm{H}=0.95 \AA]$ and were refined using a riding model, with $U_{\text {iso }}(\mathrm{H})=1.2 U_{\mathrm{eq}}(\mathrm{C})$. 


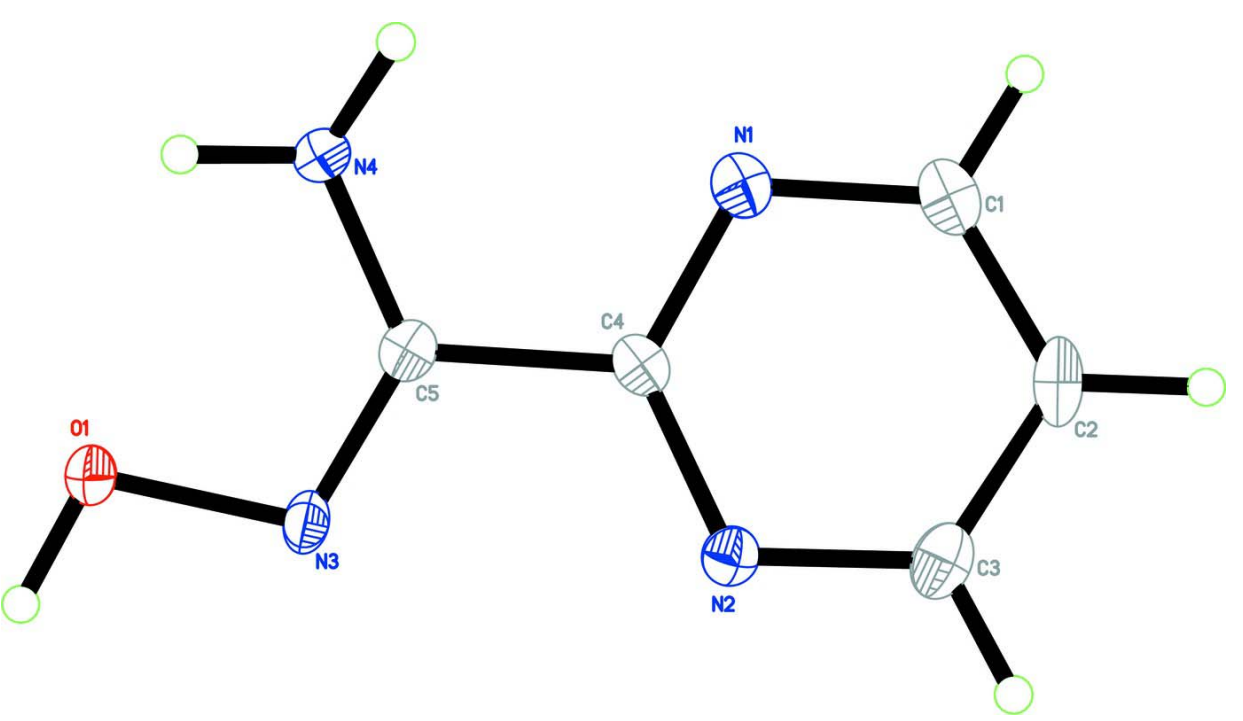

\section{Figure 1}

The molecular structure of the title compound with atom labels. Displacement ellipsoids are shown at the 50\% probability level.

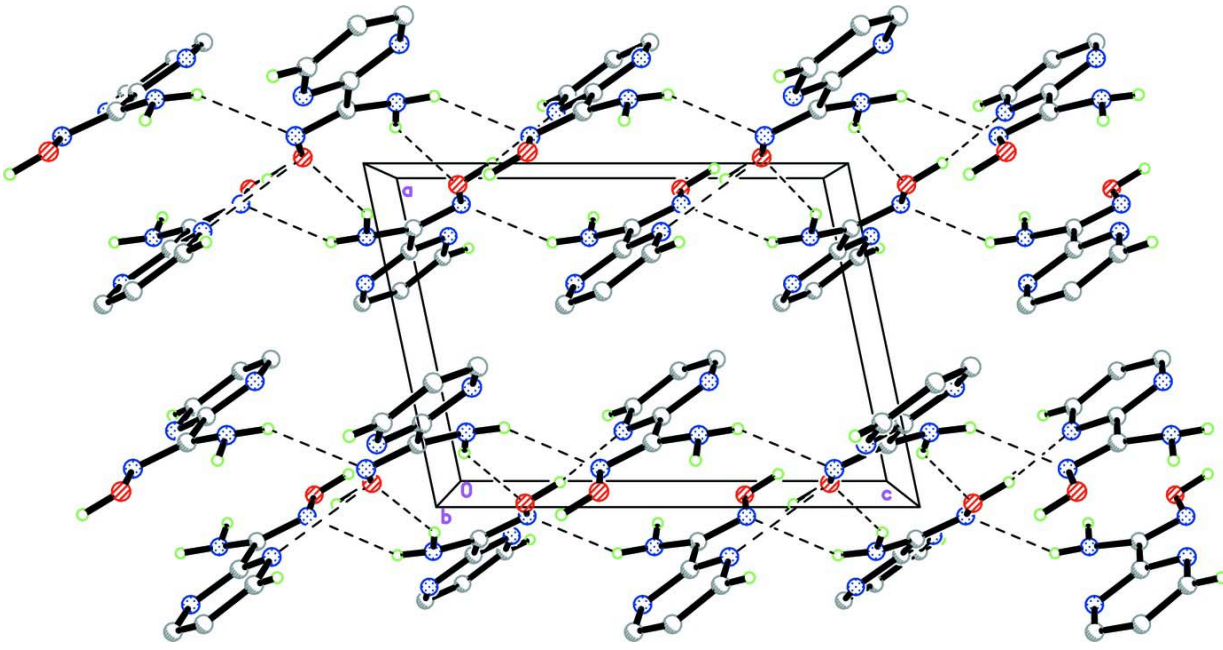

\section{Figure 2}

The crystal packing of the title compound viewed along the $b$ axis die=rection. $\mathrm{H}$ atoms not involved in intermolecular interactions (dashed lines) have been omitted for clarity.

\section{$N^{\prime}$-Hydroxypyrimidine-2-carboximidamide}

Crystal data

$\mathrm{C}_{5} \mathrm{H}_{6} \mathrm{~N}_{4} \mathrm{O}$

$M_{r}=138.14$

Monoclinic, $P 2_{1} / c$

Hall symbol: -P $2 \mathrm{ybc}$

$a=7.4066$ (7) $\AA$

$b=8.0165(8) \AA$

$c=10.2200(9) \AA$

$\beta=101.888(6)^{\circ}$
$V=593.8(1) \AA^{3}$

$Z=4$

$F(000)=288$

$D_{\mathrm{x}}=1.545 \mathrm{Mg} \mathrm{m}^{-3}$

Mo $K \alpha$ radiation, $\lambda=0.71073 \AA$

Cell parameters from 1905 reflections

$\theta=2.8-29.9^{\circ}$

$\mu=0.12 \mathrm{~mm}^{-1}$ 
$T=100 \mathrm{~K}$

Plate, colourless

\section{Data collection}

Bruker SMART APEXII CCD area-detector diffractometer

Radiation source: fine-focus sealed tube

Graphite monochromator

$\varphi$ and $\omega$ scans

Absorption correction: multi-scan

(SADABS; Bruker, 2009)

$T_{\min }=0.931, T_{\max }=0.990$

Refinement

Refinement on $F^{2}$

Least-squares matrix: full

$R\left[F^{2}>2 \sigma\left(F^{2}\right)\right]=0.054$

$w R\left(F^{2}\right)=0.181$

$S=1.13$

1030 reflections

103 parameters

0 restraints

Primary atom site location: structure-invariant direct methods
$0.62 \times 0.17 \times 0.08 \mathrm{~mm}$

4073 measured reflections

1030 independent reflections

831 reflections with $I>2 \sigma(I)$

$R_{\text {int }}=0.047$

$\theta_{\text {max }}=25.0^{\circ}, \theta_{\text {min }}=2.8^{\circ}$

$h=-8 \rightarrow 8$

$k=-9 \rightarrow 8$

$l=-12 \rightarrow 12$

Secondary atom site location: difference Fourier map

Hydrogen site location: inferred from neighbouring sites

$\mathrm{H}$ atoms treated by a mixture of independent and constrained refinement

$w=1 /\left[\sigma^{2}\left(F_{\mathrm{o}}^{2}\right)+(0.093 P)^{2}+0.7207 P\right]$ where $P=\left(F_{\mathrm{o}}^{2}+2 F_{\mathrm{c}}^{2}\right) / 3$

$(\Delta / \sigma)_{\max }<0.001$

$\Delta \rho_{\max }=0.29$ e $\AA^{-3}$

$\Delta \rho_{\min }=-0.31$ e $\AA^{-3}$

\section{Special details}

Experimental. The crystal was placed in the cold stream of an Oxford Cryosystems Cobra open-flow nitrogen cryostat operating at 100.0 (1) K.

Geometry. All e.s.d.'s (except the e.s.d. in the dihedral angle between two 1.s. planes) are estimated using the full covariance matrix. The cell e.s.d.'s are taken into account individually in the estimation of e.s.d.'s in distances, angles and torsion angles; correlations between e.s.d.'s in cell parameters are only used when they are defined by crystal symmetry. An approximate (isotropic) treatment of cell e.s.d.'s is used for estimating e.s.d.'s involving 1.s. planes.

Refinement. Refinement of $F^{2}$ against ALL reflections. The weighted $R$-factor $w R$ and goodness of fit $S$ are based on $F^{2}$, conventional $R$-factors $R$ are based on $F$, with $F$ set to zero for negative $F^{2}$. The threshold expression of $F^{2}>\sigma\left(F^{2}\right)$ is used only for calculating $R$-factors (gt) etc. and is not relevant to the choice of reflections for refinement. $R$-factors based on $F^{2}$ are statistically about twice as large as those based on $F$, and $R$ - factors based on ALL data will be even larger.

Fractional atomic coordinates and isotropic or equivalent isotropic displacement parameters $\left(\AA^{2}\right)$

\begin{tabular}{lllll}
\hline & $x$ & $y$ & $z$ & $U_{\text {iss }} * / U_{\text {eq }}$ \\
\hline O1 & $0.9616(3)$ & $0.4128(2)$ & $0.15856(18)$ & $0.0171(6)$ \\
$\mathrm{N} 1$ & $0.6515(3)$ & $0.0148(3)$ & $-0.1252(2)$ & $0.0159(6)$ \\
$\mathrm{N} 2$ & $0.8106(3)$ & $-0.0836(3)$ & $0.0883(2)$ & $0.0140(6)$ \\
$\mathrm{N} 3$ & $0.9064(3)$ & $0.2423(3)$ & $0.1497(2)$ & $0.0150(6)$ \\
$\mathrm{N} 4$ & $0.8007(3)$ & $0.3212(3)$ & $-0.0758(2)$ & $0.0155(6)$ \\
$\mathrm{C} 1$ & $0.5875(4)$ & $-0.1406(4)$ & $-0.1538(3)$ & $0.0183(7)$ \\
$\mathrm{H} 1 \mathrm{~A}$ & 0.5107 & -0.1612 & -0.2389 & $0.022^{*}$ \\
$\mathrm{C} 2$ & $0.6283(4)$ & $-0.2713(4)$ & $-0.0652(3)$ & $0.0198(7)$ \\
$\mathrm{H} 2 \mathrm{~A}$ & 0.5801 & -0.3800 & -0.0867 & $0.024 *$ \\
$\mathrm{C} 3$ & $0.7425(4)$ & $-0.2368(4)$ & $0.0560(3)$ & $0.0183(7)$ \\
$\mathrm{H} 3 \mathrm{~A}$ & 0.7743 & -0.3244 & 0.1191 & $0.022^{*}$
\end{tabular}


supporting information

\begin{tabular}{lllll}
$\mathrm{C} 4$ & $0.7592(3)$ & $0.0362(3)$ & $-0.0043(2)$ & $0.0127(6)$ \\
$\mathrm{C} 5$ & $0.8291(3)$ & $0.2095(3)$ & $0.0268(2)$ & $0.0126(7)$ \\
$\mathrm{H} 2 \mathrm{~N} 4$ & $0.869(4)$ & $0.413(4)$ & $-0.057(3)$ & $0.012(7)^{*}$ \\
$\mathrm{H} 1 \mathrm{~N} 4$ & $0.784(4)$ & $0.281(4)$ & $-0.162(3)$ & $0.025(8)^{*}$ \\
$\mathrm{H} 1 \mathrm{O} 1$ & $1.029(5)$ & $0.429(4)$ & $0.247(4)$ & $0.022(8)^{*}$ \\
\hline
\end{tabular}

Atomic displacement parameters $\left(\AA^{2}\right)$

\begin{tabular}{lllllll}
\hline & $U^{11}$ & $U^{22}$ & $U^{33}$ & $U^{12}$ & $U^{13}$ & $U^{23}$ \\
\hline O1 & $0.0221(11)$ & $0.0123(11)$ & $0.0157(11)$ & $-0.0038(8)$ & $0.0012(9)$ & $-0.0005(7)$ \\
N1 & $0.0142(12)$ & $0.0173(13)$ & $0.0169(12)$ & $-0.0003(10)$ & $0.0048(10)$ & $-0.0023(9)$ \\
N2 & $0.0130(12)$ & $0.0135(12)$ & $0.0156(12)$ & $0.0008(9)$ & $0.0033(10)$ & $0.0000(9)$ \\
N3 & $0.0164(12)$ & $0.0098(12)$ & $0.0185(12)$ & $-0.0026(10)$ & $0.0027(10)$ & $-0.0001(9)$ \\
N4 & $0.0196(13)$ & $0.0134(13)$ & $0.0130(12)$ & $-0.0015(11)$ & $0.0023(10)$ & $0.0016(9)$ \\
C1 & $0.0139(14)$ & $0.0209(15)$ & $0.0212(14)$ & $-0.0018(12)$ & $0.0058(12)$ & $-0.0060(12)$ \\
C2 & $0.0166(15)$ & $0.0129(14)$ & $0.0319(16)$ & $-0.0030(11)$ & $0.0096(13)$ & $-0.0071(12)$ \\
C3 & $0.0177(15)$ & $0.0147(14)$ & $0.0246(15)$ & $0.0015(12)$ & $0.0096(12)$ & $0.0004(11)$ \\
C4 & $0.0092(13)$ & $0.0160(15)$ & $0.0138(13)$ & $0.0006(11)$ & $0.0043(11)$ & $-0.0032(10)$ \\
C5 & $0.0099(13)$ & $0.0137(14)$ & $0.0160(13)$ & $0.0011(11)$ & $0.0065(11)$ & $0.0002(10)$ \\
\hline
\end{tabular}

Geometric parameters $\left(\AA,{ }^{\circ}\right)$

\begin{tabular}{llll}
\hline $\mathrm{O} 1-\mathrm{N} 3$ & $1.424(3)$ & $\mathrm{N} 4-\mathrm{H} 2 \mathrm{~N} 4$ & $0.89(3)$ \\
$\mathrm{O} 1-\mathrm{H} 1 \mathrm{O} 1$ & $0.94(4)$ & $\mathrm{N} 4-\mathrm{H} 1 \mathrm{~N} 4$ & $0.92(3)$ \\
$\mathrm{N} 1-\mathrm{C} 4$ & $1.336(3)$ & $\mathrm{C} 1-\mathrm{C} 2$ & $1.378(4)$ \\
$\mathrm{N} 1-\mathrm{C} 1$ & $1.343(4)$ & $\mathrm{C} 1-\mathrm{H} 1 \mathrm{~A}$ & 0.9500 \\
$\mathrm{~N} 2-\mathrm{C} 3$ & $1.343(4)$ & $\mathrm{C} 2-\mathrm{C} 3$ & $1.376(4)$ \\
$\mathrm{N} 2-\mathrm{C} 4$ & $1.347(3)$ & $\mathrm{C} 2-\mathrm{H} 2 \mathrm{~A}$ & 0.9500 \\
$\mathrm{~N} 3-\mathrm{C} 5$ & $1.295(3)$ & $\mathrm{C} 3-\mathrm{H} 3 \mathrm{~A}$ & 0.9500 \\
$\mathrm{~N} 4-\mathrm{C} 5$ & $1.362(3)$ & $\mathrm{C} 4-\mathrm{C} 5$ & $1.494(4)$ \\
& & & \\
$\mathrm{N} 3-\mathrm{O} 1-\mathrm{H} 1 \mathrm{O} 1$ & $106.1(18)$ & $\mathrm{C} 3-\mathrm{C} 2-\mathrm{H} 2 \mathrm{~A}$ & 121.6 \\
$\mathrm{C} 4-\mathrm{N} 1-\mathrm{C} 1$ & $116.0(2)$ & $\mathrm{N} 2-\mathrm{C} 3-\mathrm{C} 2$ & 121.6 \\
$\mathrm{C} 3-\mathrm{N} 2-\mathrm{C} 4$ & $116.2(2)$ & $\mathrm{N} 2-\mathrm{C} 3-\mathrm{H} 3 \mathrm{~A}$ & $122.4(3)$ \\
$\mathrm{C} 5-\mathrm{N} 3-\mathrm{O} 1$ & $108.7(2)$ & $\mathrm{C} 2-\mathrm{C} 3-\mathrm{H} 3 \mathrm{~A}$ & 118.8 \\
$\mathrm{C} 5-\mathrm{N} 4-\mathrm{H} 2 \mathrm{~N} 4$ & $112.9(18)$ & $\mathrm{N} 1-\mathrm{C} 4-\mathrm{C} 2$ & 118.8 \\
$\mathrm{C} 5-\mathrm{N} 4-\mathrm{H} 1 \mathrm{~N} 4$ & $118(2)$ & $\mathrm{N} 2-\mathrm{C} 4-\mathrm{C} 5$ & $125.9(2)$ \\
$\mathrm{H} 2 \mathrm{~N} 4-\mathrm{N} 4-\mathrm{H} 1 \mathrm{~N} 4$ & $117(3)$ & $\mathrm{N} 3-\mathrm{C} 5-\mathrm{N} 4$ & $115.6(2)$ \\
$\mathrm{N} 1-\mathrm{C} 1-\mathrm{C} 2$ & $122.8(2)$ & $\mathrm{N} 3-\mathrm{C} 5-\mathrm{C} 4$ & $125.5(2)$ \\
$\mathrm{N} 1-\mathrm{C} 1-\mathrm{H} 1 \mathrm{~A}$ & 118.6 & $\mathrm{C} 5-\mathrm{C} 4$ & $117.4(2)$ \\
$\mathrm{C} 2-\mathrm{C} 1-\mathrm{H} 1 \mathrm{~A}$ & 118.6 & $\mathrm{C} 3-\mathrm{N} 2-\mathrm{C} 4-\mathrm{C} 5$ & $117.1(2)$ \\
$\mathrm{C} 3-\mathrm{C} 2-\mathrm{C} 1$ & $116.7(3)$ & $\mathrm{O} 1-\mathrm{N} 3-\mathrm{C} 5-\mathrm{C} 5-\mathrm{C} 4$ & $179.3(2)$ \\
& & $\mathrm{N} 1-\mathrm{C} 4-\mathrm{C} 5-\mathrm{N} 3$ & $-1.7(3)$ \\
$\mathrm{C} 4-\mathrm{N} 1-\mathrm{C} 1-\mathrm{C} 2$ & $0.2(4)$ & $\mathrm{N} 2-\mathrm{C} 4-\mathrm{C} 5-\mathrm{N} 3$ & $-179.51(19)$ \\
$\mathrm{N} 1-\mathrm{C} 1-\mathrm{C} 2-\mathrm{C} 3$ & $-1.1(4)$ & $0.8(4)$ & $168.3(2)$ \\
$\mathrm{C} 4-\mathrm{N} 2-\mathrm{C} 3-\mathrm{C} 2$ & $0.5(4)$ & $-12.7(3)$ \\
$\mathrm{C} 1-\mathrm{C} 2-\mathrm{C} 3-\mathrm{N} 2$ & $1.3(4)$ & &
\end{tabular}


supporting information

$\begin{array}{llll}\mathrm{C} 1-\mathrm{N} 1-\mathrm{C} 4-\mathrm{C} 5 & -179.8(2) & \mathrm{N} 1-\mathrm{C} 4-\mathrm{C} 5-\mathrm{N} 4 & -9.7(3) \\ \mathrm{C} 3-\mathrm{N} 2-\mathrm{C} 4-\mathrm{N} 1 & -1.8(4) & \mathrm{N} 2-\mathrm{C} 4-\mathrm{C} 5-\mathrm{N} 4 & 169.3(2)\end{array}$

Hydrogen-bond geometry $\left(A,{ }^{\circ}\right)$

\begin{tabular}{lllll}
\hline$D-\mathrm{H} \cdots A$ & $D-\mathrm{H}$ & $\mathrm{H} \cdots A$ & $D \cdots A$ & $D-\mathrm{H} \cdots A$ \\
\hline $\mathrm{N} 4-\mathrm{H} 2 N 4 \cdots \mathrm{O} 1^{\mathrm{i}}$ & $0.89(3)$ & $2.27(3)$ & $2.996(3)$ & $139(3)$ \\
$\mathrm{N} 4-\mathrm{H} 1 N 4 \cdots \mathrm{N} 3^{\mathrm{ii}}$ & $0.92(3)$ & $2.30(3)$ & $3.106(3)$ & $146(3)$ \\
$\mathrm{O} 1-\mathrm{H} 1 O 1 \cdots \mathrm{N} 2^{\mathrm{iii}}$ & $0.95(4)$ & $1.85(4)$ & $2.783(3)$ & $167(3)$ \\
$\mathrm{C} 3-\mathrm{H} 3 A \cdots \mathrm{O} 1^{\text {iv }}$ & 0.95 & 2.51 & $3.305(4)$ & 141 \\
\hline
\end{tabular}

Symmetry codes: (i) $-x+2,-y+1,-z$; (ii) $x,-y+1 / 2, z-1 / 2$; (iii) $-x+2, y+1 / 2,-z+1 / 2$; (iv) $x, y-1, z$. 